\title{
Controlling Internet Queue Dynamics using Recursively Identified Models
}

\author{
Frida Gunnarsson, Fredrik Gunnarsson, Fredrik Gustafsson ${ }^{1}$ \\ Division of Communication Systems \\ Dep.of EE, Linköpings universitet \\ SE-581 83 Linköping, Sweden \\ \{frida,fred,fredrik\}@isy.liu.se
}

\begin{abstract}
Data traffic on the Internet of today is controlled by a non-linear controller (TCP) at each sender node, which increases packet transmission rate each time an acknowledgement is received in due time, and decreases otherwise. The routers may co-operate with TCP by deliberately dropping packets, so called early drops. The idea is to decrease packet arrival rate before the queue becomes full and hard drops of packets are necessary. State of the art is to compute the probability of an early drop as a static function of the (filtered) queue length.
\end{abstract}

We propose to use an auto-regressive model for the oscillative behaviour of the queue length that can be observed in practice. With this model, the queue length can be predicted and a dynamic algorithm for computing the early drop probability can be used. We suggest a very simple modification of existing algorithms, where a short-time prediction is used instead of the current queue value, and demonstrate using $n s-2$ simulations that the overall throughput increases.

\section{Introduction}

Internet performance is limited by the capacity of packet queues in routers. Traffic control protocols, TCP, estimate the available capacity on the link between the corresponding sender and receiver and using this decide on the senders' data rate. TCP decreases transmission rate if a packet is lost (no acknowledgement is received in due time) and increases it when a packet is successfully delivered (acknowledgement received).

During the last decade, numerous suggestions for improving TCP performance have appeared, Low et al.

\footnotetext{
${ }^{1}$ This work is supported by the Swedish Agency for Innovation Systems, VINNOVA, http://www.vinnova.se, and the competence center ISIS (Information Systems for Industrial Control and Supervision), Linköpings universitet, http://www.control.isy.liu.se/isis/, which all are acknowledged.
}

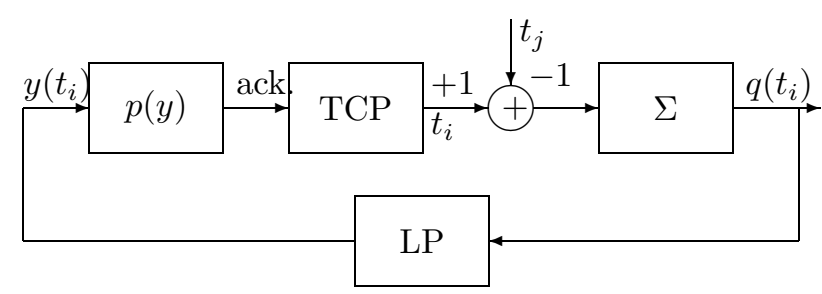

Figure 1: The basic Internet feedback loop: Each sender has TCP flow control, which increases data rate for each acknowledgement (ack) and decreases it rapidly when no ack is received. This ack is the only feedback variable from the network, but the router can co-operate with TCP in a constructive manner by dropping packets early, and therefore stopping the arrival of acks. This is in RED done by low-pass filtering the observed queue length $y(t)=H(q) q(t)$ and using a static table for selecting dropping probability $0 \leq p(y) \leq 1$.

(2002). However, dropped packets inevitably decrease traffic and since TCP does not allow more feedback than acknowledgements, there is no way TCP can know when the queues are almost full. For that reason, an interesting idea has been suggested: accept this limited feedback information, and decentralize the control structure, so the routers on the way cooperate with the senders' TCP Floyd and Jacobson (1993). The basic idea is to deliberately drop packets from intermediate queues on the link before actual overflow, so called early drops. This may reduce the number of ordinary, or hard drops, and also reduce the oscillations in the queue length. Oscillations have a tendency to increase in amplitude until the queue saturates, which inevitably decreases performance, so a stabilizing controller is wanted.

One of the questions regarding this problem is on what grounds we should perform the early drops. An intuitive way is to relate the drops to the queue length and the first proposal, in Floyd and Jacobson (1993), introduces a drop probability proportional to the queue length, which decides whether or not a newly arrived 
packet is dropped or received. This strategy is called Random Early Detection, RED, and it is currently employed in routers worldwide. This procedure is illustrated in Figure 1. The queue length $q\left(t_{i}\right)$ observed at each packet arrival time $t_{i}$ (but not when a packet leaves the queue at time $t_{j}$ ) is first low-pass filtered to get a smoother measure of queue length $y\left(t_{i}\right)$. A standard router drops a packet when the queue is full and forwards it otherwise. In RED, a packet is dropped with probability $p(y)$ when the queue is almost full, see Figure 4 for an example. A late or missing ack forces TCP in the sender to rapidly decrease its transmission rate, so the packet arrival events $t_{i}$ come at a slower rate.

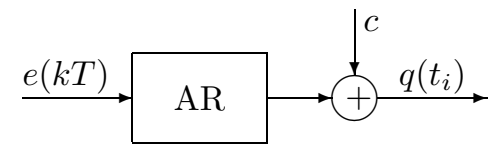

Figure 2: Model of the feedback loop in Figure 1. This is used to predict $y\left(t_{i}+n T\right)$, and RED is modified to use $p\left(y\left(t_{i}+n T\right)\right)$ which essentially implements a derivative control action.

Performance is improved in RED compared to ordinary queues but tuning of internal parameters have caused large problems. Practice also shows that the queue lengths still oscillate, although somewhat less than without RED. Due to these drawbacks, several proposals have been presented to improve RED. Most of them are based on the same structure but with different dropping decision mechanisms: The dropping probability is calculated based on packet losses and idle queue time, BLUE, Feng et al. (1999); A PI controller is tuned based on a simplified physical model, Hollot et al. (2001); A virtual queue is used to decide the drops, AVQ, Kunniyur and Srikant (2001), and SVB, Deng et al. (2002). A nice comparison of a few algorithms can be found in Zhu et al. (2002)

The interplay of routers, the multitude of non-linear TCP controllers and random feedback delays is very hard to model. However, non-linear dynamics and feedback delays are known to cause oscillations, which as mentioned before can also be observed in practice. For that reason, we suggest to use a time-varying autoregressive $(\mathrm{AR})$ queue model with a time-varying mean level $c$, as illustrated in Figure 2. The advantage is that this model can predict how the queue length will evolve in the near future, and we can design a more clever dropping mechanism. There are many possibilities of model-based control. However, as a first attempt to improve performace and to get an algorithm that fits the protocols of today, we investigate a very simple but yet natural modification of RED. We replace the block $p\left(y\left(t_{i}\right)\right)$, but otherwise keep everything intact in Fig- ure 1 , with $p\left(\hat{y}\left(t_{i}+T_{\text {pred }}\right)\right)$. This could be interpreted as replacing a non-linear proportional controller with a non-linear proportional and derivative controller, that reacts with more drops if the queue size is likely to increase as is the case in Figure 3.

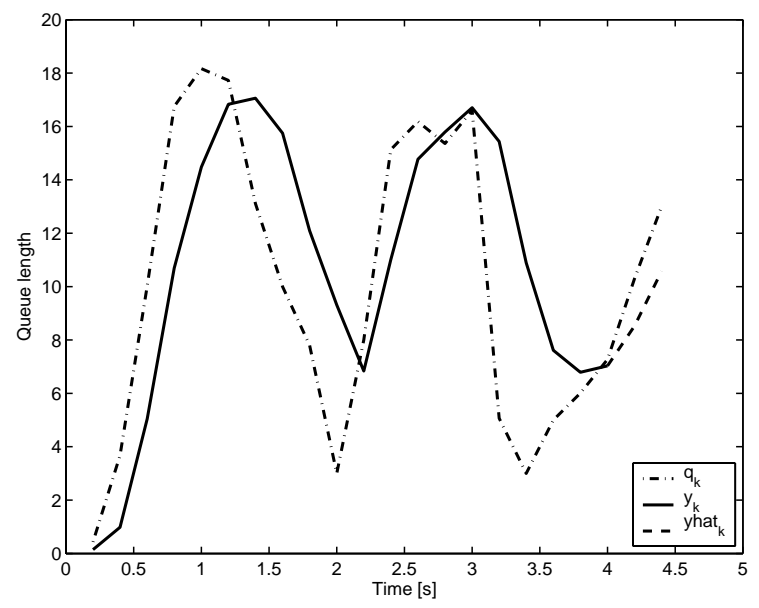

Figure 3: The filtered queue length (solid) augmented with the on-line estimated AR model predictions at time $4 \mathrm{~s}$, and the original queue length (dash-dotted).

The outline is as follows. Section 2 summarizes the RED algorithm. Then the method of performance evaluation is presented in Sec. 3. The theory and choices for modeling and control are presented in Sec. 4. In Sec. 5 the results for both the recursive identification and the control of the queue dynamics is shown and Sec. 6 concludes with a discussion about achievements and challenges.

\section{The RED algorithm}

Since the design is based on minor modifications of the existing algorithm RED, we describe its functionality briefly. Upon a packet arrival at time, $t_{i}$, the current queue length, $q\left(t_{i}\right)$ is measured, and the average queue length, $y\left(t_{i}\right)$, is calculated,

$$
y\left(t_{i}\right)= \begin{cases}\left(1-w_{q}\right)^{m} y\left(t_{i-1}\right) & q\left(t_{i}\right)=0 \\ \left(1-w_{q}\right) y\left(t_{i-1}\right)+w_{q} q\left(t_{i}\right) & q\left(t_{i}\right)>0\end{cases}
$$

with $m=$ constant $\cdot\left(t_{i}-t_{e}\right)$ and $t_{e}$ is the time when the queue became empty. $w_{q}$ is a weight factor, between 0 and 1 , to control the impact of new measurements. This can be interpreted as a first order low-pass filter (forgetting factor filter) with a special fast decaying mode when the queue is empty. Next, a dropping probability, $p$, is calculated

$$
p\left(y\left(t_{i}\right)\right)= \begin{cases}0 & y\left(t_{i}\right) \leq m_{q} \\ 1 & y\left(t_{i}\right) \geq M_{q} \\ M_{p} \frac{y\left(t_{i}\right)-m_{q}}{M_{q}-m_{q}} & \text { otherwise }\end{cases}
$$


Fig. 4 shows an example of $p(y)$ for RED. $p(y)$ is also called the dropping probability profile.

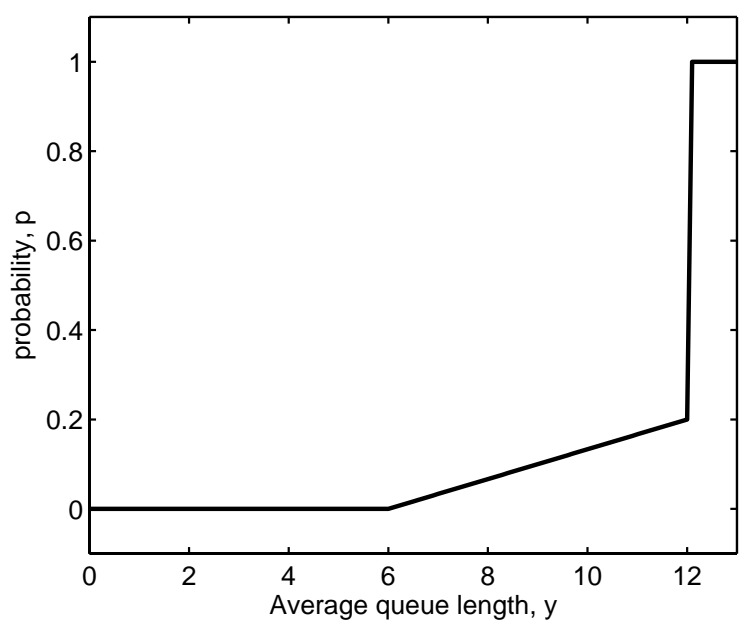

Figure 4: RED's drop probability, when $m_{q}=6, M_{q}=12$ and $M_{p}=0.2$.

In Fig. 3 a segment of the filtered queue length, $y$, during a certain simulation setup is shown. The queue weight, $w_{q}$, was set to 0.05 , the true queue length, $q$, is dash-dotted. It is well known that in order to damp oscillations a proportional derivating, PD, controller can be used. To be able to do this, an accurate estimate of the derivative is needed, i.e., the signal needs to be filtered in some way. Since the signal locally behaves as a self-oscillating process, finding the basic frequency provides a tool to calculate the derivative. This can be done by adapting a autoregressive model to data, after it has been low pass-filtered. Considerations on how to design the low-pass filter and on how to decide the model structure have to be made. The following sections will discuss these matters.

\section{Performance measurements}

It is maybe not obvious what to consider when evaluating different control schemes for an Internet queue. As was discussed previously in Gunnarsson et al. (2002) the goal is basically the same as for any control system: a fast response to sudden load changes, good suppression of disturbances and a stable steady state level. In Internet queue terms this means to decrease the oscillations and keep the queue length at some nominal level, below maximum to reduce the number of hard drops and above zero to keep the queue fully utilized. As mentioned before, focus is on characterizing the oscillations in this work. To measure performance frequency analysis will be used, as well as the total throughput for different control schemes.

\section{Adaptive filtering and RED modification}

This work will both investigate the use of system identification and simple control methods as a means to improve performance in Internet queues. Now, the choices in the design are described.

\subsection{Modeling}

The queue length shows an oscillative behaviour, the example in Fig. 3 is typical. Let $z$ describe the oscillating part. One possible model structure is an autoregressive model

$$
z(t)+a_{1} z(t-T)+a_{2} z(t-2 T)+a_{3} z(t-2 T)=e(t) .
$$

Two of the parameters describe the oscillations as a sinusoid locally and the third one is used to take into account the dynamics of the low-pass filter, so one pole in the estimated model is likely to occur at the exponential filter pole $w_{q}$ in Eq. (1). The connection between $w_{q}$ and the $a_{i}$ 's is most clear if $T_{i}=t_{i}-t_{i-1}=n T$. The level is described by adding a constant, $c$, to $z(t)$ which gives:

$$
\begin{aligned}
y(t)= & z(t)+c \\
= & -a_{1}(y(t-T)-c)+ \\
& -a_{2}(y(t-2 T)-c)+ \\
& -a_{3}(y(t-3 T)-c)+c+e(t) \\
= & -a_{1} y(t-T)-a_{2} y(t-2 T)+ \\
& -a_{3} y(t-3 T)+b+e(t) \\
= & \varphi(t)^{T} \theta+e(t)
\end{aligned}
$$

where

$$
\begin{aligned}
b & =\left(a_{1}+a_{2}+a_{3}+1\right) c \\
\varphi(t) & =(-y(t-T),-y(t-2 T),-y(t-3 T), 1)^{T} \\
\theta(t) & =\left(a_{1}, a_{2}, a_{3}, b\right)^{T}
\end{aligned}
$$

\subsection{Adaptive filtering}

For the implementation of the recursive identification procedure Recursive Least Squares, RLS, is chosen. This has a fairly easy implementation with nice convergence properties. The Least Mean Square, LMS, algorithm was also considered but was shown to have severe convergence problems and the normalized version, NLMS, failed to identify the true parameters, and instead let the level, $c$, track the measurements, $y$. More theoretical properties for different recursive identification algorithms, see (Ljung, 1999, Ch. 11). 


\section{Algorithm 1 (RLS)}

For each update step the following is performed:

Let $\theta(t-1)$ be the estimate of $\theta$ at time $t-1$. We also store an internal matrix $P$ for each time instant. Calculate a new estimate at time $t$ according to

$$
\begin{aligned}
\hat{y}(t)= & \varphi(t)^{T} \theta(t-1) \\
\varepsilon(t)= & y(t)-\hat{y}(t) \\
P(t)= & \frac{1}{\lambda}(P(t-1)+ \\
& \left.-\frac{P(t-1) \varphi(t) \varphi^{T}(t) P(t-1)}{\lambda+\varphi^{T}(t) P(t-1) \varphi(t)}\right) \\
K(t)= & P(t) \varphi(t) \\
\theta(t)= & \theta(t-1)+K(t) \varepsilon(t)
\end{aligned}
$$

Here $\lambda$ is the forgetting factor chosen as less than but close to one.

Since it is not likely that one of the update times $t_{i}$ matches $t-n T, n=1,2,3$, simple linear interpolation between the two surrounding measurement points is used whenever an old value of $y$ is needed. The question arises what the suitable $T$ is. Using the estimated model, the oscillating frequency, $f$, can be found and $T$ should then, as a rule of thumb, be chosen as a tenth of the period, i.e., $T \approx \frac{1}{f} \frac{1}{10}$. To begin with a time constant is chosen from inspection and later investigations have to solve the problem of automation.

\subsection{Frequency estimation}

An AR-model will try to describe the dominating frequencies in the signal. The frequencies can be found looking at the angles for the complex roots of the characteristic polynomial, i.e, the $x$ solving $x^{3}+a_{1} x^{2}+a_{2} x+$ $a_{3}=0$. The frequencies are then

$$
f=\frac{\operatorname{angle}(x)}{\pi} \frac{1}{2 T}
$$

and in the case of three parameters the model is able to describe one frequency; the fundamental oscillation mode.

\subsection{Non-parametric D action}

In standard RED, the packet dropping probability is calculated and the packet is then dropped or accepted based on this probability. A natural extension is to only drop packets when the queue is increasing, which is the simplest possible inclusion of derivative action in the controller. A non-parametric estimate of the derivative is simply estimated from the filtered queue length as illustrated in Fig. 5

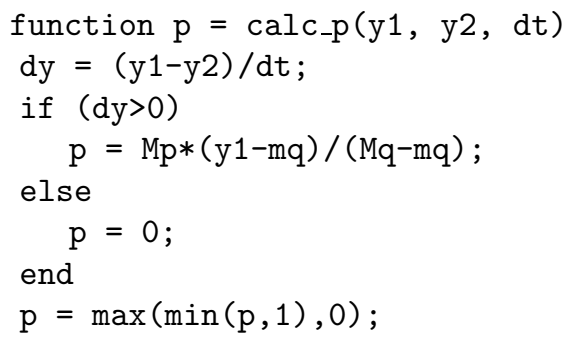

Figure 5: In all simulations, $M_{q}=$ Mq was set to the actual queue length, $m_{q}=$ mq was half the total queue length and $M_{p}=$ Mp was 0.2 .

\subsection{Model-based prediction}

The idea with the adaptively estimated AR model is to predict the evolution of the near future queue length. This is done in the simplest possible way by just replacing $y(t)$ with $\hat{y}\left(t+T_{\text {pred }}\right)$ at each instance it occurs. Thus, we can also combine the non-parametric PD-controller with predictions.

\subsection{Summary of control strategies}

Combining prediction and D action to the basis RED algorithm gives four alternatives, which all can be implementing using the function in Fig. 5: Here y means the current measurement, y_old the previous one, y_hat means the estimated value of $y\left(t+T_{\text {pred }}\right)$, $t$ is the current time, $t_{-}$old the time of the previous measurement and $\mathrm{T}$ is the prediction horizon and the time constant of the model. $\mathrm{p}$ is the calculated dropping probability. The generic algorithm in Section 4.4 can be used for all four possibilities.

\section{Basic RED}

$\mathrm{p}=\mathrm{cal}_{-} \mathrm{p}(\mathrm{y}, 0, \mathrm{y})$ Ordinary RED, where only the current measurement is considered.

\section{RED with prediction}

$$
\mathrm{p}=\text { calc }-\mathrm{p} \text { (y_hat, } 0, \mathrm{y} \text { _hat }) \quad \text { The RED- }
$$
functionality is applied to the predicted value.

\section{RED with $D$ action}

$\mathrm{p}=\mathrm{cal}_{-} \mathrm{p}\left(\mathrm{y}, \mathrm{y}_{-} \mathrm{old}, \mathrm{t}-\mathrm{t}_{-} \mathrm{old}\right) \mathrm{A}$ positive value of the dropping probability is used only if the estimate of the derivative is positive, i.e., the queue length increases.

\section{RED with $\mathrm{D}$ action and prediction}

$\mathrm{p}=$ calc_p $\left(\mathrm{y} \_\right.$hat $\left., \mathrm{y}, \mathrm{T}\right)$ As above but with the predicted value instead of the measured.

\section{Results}

The Network simulator, NS (n.d.), is used for evaluation. Implementing a special version of the RED queue, 
the effects of both identification and control can be studied. Only one queue is studied, which experiences a varying number of transmission flows. For comparative studies, the the start and stop times for the flows are fixed in each simulation to get the comparable and reproducible conditions for all controllers.

Comparing this with the frequency spectrum of the signal will show if the model is good at finding the correct frequency. Studying the result shows that the frequency changes over time in the simulation, and we concentrate on a segment where the frequency estimate was fairly constant. Fig. 6 shows the estimated frequency and the spectrum of the signal for two different time intervals. It is clear that several frequencies are present in the signal during these intervals, the output form the ARestimation is the highest of the dominating ones, i.e., the fastest one of the larger variations are followed.
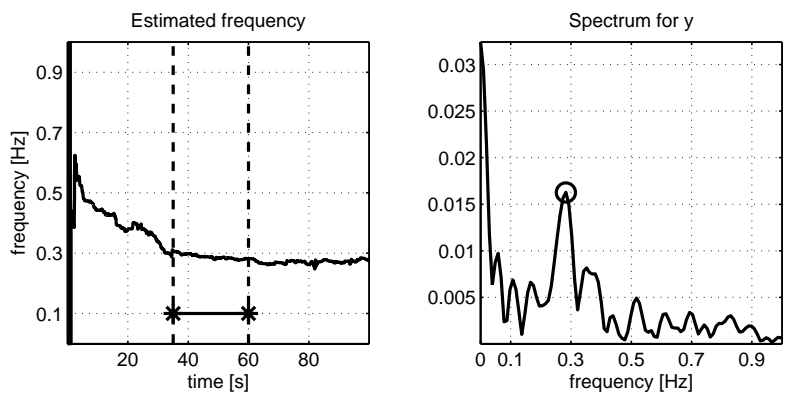

Figure 6: Evaluating the methods ability to find the correct frequency. To the right are the spectrum over time interval indicated in the picture to the left. The captured peak is circled.

The time constant of the system is important and we need to carefully consider how to adapt it when needed. Simulations not shown indicate that it is the send rate of the queue that is the main cause of changing time constant. Here, the scenarios have been adapted to work for $T=0.2 \mathrm{~s}$, the results are valid no matter what value we choose but the choice is still a design question. To improve performance automatic detection of an accurate time constant should be considered.

A few performance measures is summarized in Tab. 1. These numbers are calculated for one single run with the exact same setup for all four controllers. The following observations can be made from Tab. 1:

- Performance (number of sent packets, throughput) is improved by increasing the total number of drops.

- Prediction can improve the throughput considerably, both in basic RED and with RED with D action.
Table 1: Comparison of different control schemes, see Sec. 4.6 for a description.

\begin{tabular}{|lcc|}
\hline Type & $\begin{array}{c}\text { Drops } \\
\text { early }+ \text { hard }=\text { tot }\end{array}$ & $\begin{array}{c}\text { Sent } \\
\text { unique packets }\end{array}$ \\
\hline 1 & $55+250=305$ & 3260 \\
2 & $66+247=313$ & 3569 \\
3 & $82+207=289$ & 3126 \\
4 & $62+235=297$ & 3342 \\
\hline
\end{tabular}

- The non-parametric estimate of queue derivative is not that reliable, and sometimes even decreases overall throughput.

\section{Conclusions}

Internet queues often show an oscillative behaviour caused by non-linear feedback loops in combination with time-delays. Oscillations cause regular queue saturations, where a full queue means lost packets and an empty queue implies decreased throughput to the next router. For that reason, we have studied how the queue length can be stabilized by including derivative action in the state of the art non-linear proportional controller. The simplest way is to estimate the derivative of the queue length directly. However, we have found that a model-based approach where the model predicts the queue length has high potential to increase overall throughput.

The dynamics in an Internet queue can be well described with a time-varying AR-model including a timevarying bias. The model can be estimated recursively at packet arrivals, using an adaptive filter. It has been shown in network simulations that it is possible to improve the performance by replacing the current queue length value with a model-based prediction.

\section{References}

Deng, X., S. Yi, G. Kesidis and C. Das (2002). Stabilized virtual buffer (svb) - an active queue management scheme for internet quality-of-service. In: Proc. IEEE Globecom.

Feng, W., D. Kandlur, D. Saha and Kang G. Shin (1999). BLUE: A new class of active queue management algorithms. Technical Report CSE-TR-387-99.

Floyd, Sally and Van Jacobson (1993). Random Early Detection gateways for Congestion Avoidance. IEEE/ACM Transactions on Networking 1(4), 397413.

Gunnarsson, Frida, Fredrik Gunnarsson and Fredrik Gustafsson (2002). Issues on Performance Measure- 
ments of TCP. In: Radiovetenskap och Kommunikation '02, RVK'02.

Hollot, C. V., Vishal Misra, Don Towsley and Wei-Bo Gong (2001). On designing improved controllers for aqm routers supporting tcp flows. In: INFOCOM 2001. Proceedings. IEEE. Vol. 3.

Kunniyur, S. and R. Srikant (2001). Analysis and design of an adaptive virtual queue algorithm for active queue management.. In: Proc. ACM Sigcomm.

Ljung, Lennart (1999). System Identification - Theory for the User, 2nd edition.. Prentice Hall.

Low, Steven H, Fernando Paganini and John C Doyle (2002). Internet congestion control. IEEE Control Systems Magazine pp. 28-43.

NS (n.d.). UCB/LBNL/VINT Network Simulator, ns2. Available via http://www.isi.edu/nsnam/ns/.

Zhu, Chengyu, Oliver W. W. Yang, Lames Aweya, Michel Ouellette and Delfin Y. Montuno (2002). A comparison of active queue management algorithms using the opnet modeler. IEEE Communications Magazine. 